\title{
Is lactate clearance a good mortality predictor in sepsis?(Letter)
}

- Ascarruz-Asencios, J.J. ${ }^{a} E m a i l$ Author,

- Castaneda-Montenegro, $\mathrm{G}^{\mathrm{a}}$,

- Carreazo, N.Y. ${ }^{a b}$

- View Correspondence (jump link)

- $\quad$ aEscuela de Medicina, Universidad Peruana de Ciencias Aplicadas, Lima, Peru

- $\quad$ bervicio de Cuidados Intensivos, Hospital de Emergencias Pediátricas, Lima, Peru

Abstract_View references (5)

[No abstract available]

- ISSN: 08839441

- CODEN: JCCAE

- Source Type: Journal

- Original language: English

- DOI: $10.1016 / j . j c r c .2017 .02 .003$

- Document Type: Letter

- Publisher: W.B. Saunders 